\title{
COMPLETELY INDEPENDENT SPANNING TREES IN (PARTIAL) $k$-TREES
}

\author{
Masayoshi Matsushita ${ }^{1}$, Yota Otachi ${ }^{2}$ \\ AND \\ TORU Araki ${ }^{1}$ \\ 1 Department of Computer Science \\ Gunma University, Kiryu \\ Gunma 376-8515 Japan \\ ${ }^{2}$ School of Information Science \\ Japan Advanced Institute of Science and Technology \\ Asahidai 1-1, Nomi, Ishikawa 923-1292, Japan \\ e-mail: otachi@jaist.ac.jp \\ arakit@gunma-u.ac.jp
}

\begin{abstract}
Two spanning trees $T_{1}$ and $T_{2}$ of a graph $G$ are completely independent if, for any two vertices $u$ and $v$, the paths from $u$ to $v$ in $T_{1}$ and $T_{2}$ are internally disjoint. For a graph $G$, we denote the maximum number of pairwise completely independent spanning trees by $\operatorname{cist}(G)$. In this paper, we consider $\operatorname{cist}(G)$ when $G$ is a partial $k$-tree.

First we show that $\lceil k / 2\rceil \leq \operatorname{cist}(G) \leq k-1$ for any $k$-tree $G$. Then we show that for any $p \in\{\lceil k / 2\rceil, \ldots, k-1\}$, there exist infinitely many $k$-trees $G$ such that $\operatorname{cist}(G)=p$. Finally we consider algorithmic aspects for computing $\operatorname{cist}(G)$. Using Courcelle's theorem, we show that there is a linear-time algorithm that computes $\operatorname{cist}(G)$ for a partial $k$-tree, where $k$ is a fixed constant.
\end{abstract}

Keywords: completely independent spanning trees, partial $k$-trees.

2010 Mathematics Subject Classification: 05C05, 05C38.

\section{REFERENCES}

[1] T. Araki, Dirac's condition for completely independent spanning trees, J. Graph Theory 77 (2014) 171-179.

doi:10.1002/jgt.21780 
[2] S. Arnborg and A. Proskurowski, Linear time algorithms for NP-hard problems restricted to partial $k$-trees, Discrete Appl. Math. 23 (1989) 11-24.

doi:10.1016/0166-218X(89)90031-0

[3] H.L. Bodlaender, A partial k-arboretum of graphs with bounded treewidth, Theoret. Comput. Sci. 209 (1998) 1-45.

doi:10.1016/S0304-3975(97)00228-4

[4] H.L. Bodlaender, F.V. Fomin, P.A. Golovach, Y. Otachi and E.J. van Leeuwen, Parameterized complexity of the spanning tree congestion problem, Algorithmica 64 (2012) 85-111.

doi:10.1007/s00453-011-9565-7

[5] H.L. Bodlaender and A.M.C.A. Koster, Combinatorial optimization on graphs of bounded treewidth, Comput. J. 51 (2008) 255-269. doi:10.1093/comjnl/bxm037

[6] B. Courcelle, The monadic second-order logic of graphs III: tree-decompositions, minors and complexity issues, Theor. Inform. Appl. 26 (1992) 257-286.

[7] T. Hasunuma, Completely independent spanning trees in the underlying graph of a line digraph, Discrete Math. 234 (2001) 149-157. doi:10.1016/S0012-365X(00)00377-0

[8] T. Hasunuma, Completely independent spanning trees in maximal planar graphs in: Proceedings of 28th Graph Theoretic Concepts of Computer Science (WG 2002), LNCS 2573, Springer-Verlag Berlin (2002) 235-245.

doi:10.1007/3-540-36379-3_21

[9] T. Hasunuma and C. Morisaka, Completely independent spanning trees in torus networks, Networks 60 (2012) 59-69.

doi: $10.1002 /$ net.20460

[10] P. Hliněný, S. Oum, D. Seese and G. Gottlob, Width parameters beyond tree-width and their applications, Comput. J. 51 (2008) 326-362.

doi:10.1093/comjnl/bxm052

[11] F. Péterfalvi, Two counterexamples on completely independent spanning trees, Discrete Math. 312 (2012) 808-810.

doi:10.1016/j.disc.2011.11.015

Received 24 April 2014

Revised 8 August 2014

Accepted 8 August 2014 